Acta Crystallographica Section D

Biological

Crystallography

ISSN 0907-4449

Daniel E. Holloway, Gayatri B. Chavali, $\neq$ Michelle C. Hares, Vasanta Subramanian and

\section{K. Ravi Acharya*}

Department of Biology and Biochemistry, University of Bath, Claverton Down,

Bath BA2 7AY, England

₹ Present address: Cambridge Institute of Medical Research, MRC/Wellcome Trust Building, Cambridge CB2 2XY, England.

Correspondence e-mail: k.r.acharya@bath.ac.uk

\title{
Structure of murine angiogenin: features of the substrate- and cell-binding regions and prospects for inhibitor-binding studies
}

Angiogenin is an unusual member of the pancreatic ribonuclease superfamily that induces blood-vessel formation and is a promising anticancer target. The three-dimensional structure of murine angiogenin (mAng) has been determined by X-ray crystallography. Two structures are presented: one is a complex with sulfate ions ( $1.5 \AA$ resolution) and the other a complex with phosphate ions (1.6 $\AA$ resolution). Residues forming the putative $\mathrm{B}_{1}, \mathrm{P}_{1}$ and $\mathrm{B}_{2}$ subsites occupy positions similar to their hAng counterparts and are likely to play similar roles. The anions occupy the $\mathrm{P}_{1}$ subsite, sulfate binding conventionally and phosphate adopting two orientations, one of which is novel. The $\mathrm{B}_{1}$ subsite is obstructed by Glu116 and Phe119, with the latter assuming a less invasive position than its hAng counterpart. Hydrophobic interactions between the C-terminal segment and the main body of the protein are more extensive than in hAng and may underly the lower enzymatic activity of the murine protein. Elsewhere, the structure of the H3-B2 loop supports the view that hAng Asn61 interacts directly with cell-surface molecules and does not merely stabilize adjacent regions of the hAng structure. mAng crystals appear to offer small-molecule inhibitors a clear route to the active site and may even withstand a reorientation of the $\mathrm{C}$-terminal segment that provides access to the cryptic $B_{1}$ subsite. These features represent considerable advantages over crystalline hAng and bAng.

\section{Introduction}

Angiogenin (Ang) is an angiogenic protein originally isolated from the conditioned medium of HT-29 human colon adenocarcinoma cells (Fett et al., 1985). It plays a role in tumour angiogenesis (Olson et al., 1995, 2001, 2002; Kao et al., 2002) and its increased expression has been correlated with diverse cancers in more than 20 clinical studies to date (see Kao et al., 2002). Antagonists of Ang inhibit the establishment, progression and metastasis of human tumour xenografts in athymic mice (Olson et al., 1995, 2001, 2002; Kao et al., 2002), identifying it as a promising target for anticancer therapy.

Human Ang (hAng) has been the subject of intensive functional and structural analyses. It is a member of the pancreatic ribonuclease superfamily (Kurachi et al., 1985; Strydom et al., 1985) and possesses a weak ribonucleolytic activity that is essential for angiogenicity (Shapiro et al., 1989; Shapiro \& Vallee, 1989; Curran, Shapiro \& Riordan, 1993). Its three-dimensional structure (Acharya et al., 1994; Lequin et al., 1997; Leonidas, Shapiro, Allen et al., 1999) is broadly similar to that of bovine pancreatic ribonuclease A (RNase A; EC 3.1.27.5), with which it shares $33 \%$ sequence identity. Engineered hAng variants have enabled the identification of
Received 9 August 2005

Accepted 18 September 2005

PDB References: mAng- $\mathrm{SO}_{4}$, 2bwk, r2bwksf; mAng- $\mathrm{PO}_{4}$, 2bwl, r2bwlsf. 
structural elements that govern its characteristic cell binding (Harper \& Vallee, 1989; Hallahan et al., 1991, 1992; Shapiro \& Vallee, 1992; Holloway et al., 2002), nuclear translocation (Moroianu \& Riordan, 1994) and enzymatic (Harper \& Vallee, 1988, 1989; Shapiro et al., 1989; Shapiro \& Vallee, 1989; Curran, Shapiro \& Riordan, 1993; Curran, Shapiro, Riordan et al., 1993; Russo et al., 1994, 1996; Shapiro, 1998; Leonidas, Shapiro, Allen et al., 1999; Holloway et al., 2002, 2004; Leonidas et al., 2002) activities. Once it reaches the nucleus, Ang stimulates rRNA transcription, playing a key role in endothelial cell proliferation that is necessary for angiogenesis induced by other angiogenic factors (Kishimoto et al., 2005; Tsuji et al., 2005).

Despite yielding much information, study of the human protein has limitations. Firstly, there are obvious practical difficulties in studying the role of hAng in healthy living subjects. Secondly, in all known crystal forms of hAng variants, key residues in the ribonucleolytic active site are intimately involved in crystal packing (Leonidas et al., 2001). This has prevented visualization of the binding of known lowmolecular-weight substrates and inhibitors, in turn hampering the rational design of potential anticancer agents. It has also obscured the precise nature of the change in protein conformation that is believed to accompany the binding of pyrimidine nucleotides (Russo et al., 1994). The crystal structure of bovine Ang (bAng; Acharya et al., 1995) has proved to have similar limitations.

The murine Ang (mAng) system may offer solutions to the experimental problems outlined above. The $m A n g$ gene shares $76 \%$ sequence identity with hAng (Bond \& Vallee, 1990) and is known to have comparable angiogenic activity and approximately one-third of its enzymatic activity (Nobile et al., 1996). In addition, the murine genome encodes five additional Ang-like proteins that appear to have undergone positive selection (Brown et al., 1995; Fu \& Kamps, 1997; Strydom, 1998; Cho et al., 2005). At least one of these is angiogenic (Fu et al., 1999), but others may have different functions (Nobile $e t$ al., 1996; Cho et al., 2005). Established protein-engineering and murine gene-disruption techniques provide the means for probing the physiological roles of these proteins, while the continued use of structural biology techniques will be essential for the validation and interpretation of such experiments. As a first step along this route, we present here two X-ray crystal structures of mAng. We assess their similarity to the structure of hAng, focusing on regions involved in RNA cleavage and cell binding. We also examine the suitability of these crystals for the visualization of small-molecule inhibitor binding.

\section{Materials and methods}

\subsection{Protein preparation}

mAng was prepared from the soluble extract of recombinant Escherichia coli W3110 by cation-exchange chromatography on SP-Sepharose and Mono-S media as described previously (Shapiro \& Vallee, 1992; Nobile et al., 1996). The sample was mixed with 0.25 volumes of $1 M$ potassium phos-
Table 1

Crystallographic statistics.

Values in parentheses are for the outermost resolution shell (1.55-1.50 and 1.66-1.60 A for $\mathrm{mAng}-\mathrm{SO}_{4}$ and $\mathrm{mAng}-\mathrm{PO}_{4}$, respectively).

\begin{tabular}{|c|c|c|}
\hline & $\mathrm{mAng}-\mathrm{SO}_{4}$ & $\mathrm{mAng}-\mathrm{PO}_{4}$ \\
\hline \multicolumn{3}{|l|}{ Diffraction data } \\
\hline Space group & $P 2_{1} 2_{1} 2$ & $P 2_{1} 2{ }_{1} 2$ \\
\hline Unit-cell parameters $(\AA)$ & $\begin{array}{l}a=63.8, b=56.3 \\
\quad c=35.3\end{array}$ & $\begin{array}{c}a=63.6, b=56.7, \\
\quad c=35.7\end{array}$ \\
\hline Resolution range $(\AA)$ & $40-1.5$ & $40-1.6$ \\
\hline \multicolumn{3}{|l|}{ No. of reflections } \\
\hline Measured & 130540 & 96889 \\
\hline Unique & 20302 & 16648 \\
\hline$R_{\mathrm{sym}}^{\dagger} \dagger$ & $0.068(0.224)$ & $0.067(0.425)$ \\
\hline$I / \sigma(I)$ & $17.0(4.8)$ & $16.2(4.0)$ \\
\hline Completeness (\%) & $96.3(83.4)$ & $94.1(61.4)$ \\
\hline \multicolumn{3}{|l|}{ Refined model } \\
\hline No. of reflections used & 19236 & 15772 \\
\hline$R_{\text {cryst }}$ 丰 & 0.179 & 0.177 \\
\hline \multirow{2}{*}{\multicolumn{3}{|c|}{$\begin{array}{l}\Lambda_{\text {free }} \\
\text { No. of atoms }\end{array}$}} \\
\hline & & \\
\hline Protein & 893 & 899 \\
\hline Anion & 10 & 10 \\
\hline Water & 108 & 80 \\
\hline \multicolumn{3}{|c|}{ Deviations from ideality (r.m.s.) } \\
\hline Bond lengths $(\AA)$ & 0.015 & 0.019 \\
\hline Bond angles $\left({ }^{\circ}\right)$ & 1.62 & 1.64 \\
\hline \multicolumn{3}{|c|}{ Mean $B$ factor by atom type $\left(\AA^{2}\right)$} \\
\hline Protein & 16.6 & 17.3 \\
\hline Anion & 32.4 & 27.8 \\
\hline Water & 35.8 & 34.9 \\
\hline
\end{tabular}

$\dagger R_{\mathrm{sym}}=\sum_{h} \sum_{i}\left[\left|I_{i}(h)-\langle I(h)\rangle\right| / \sum_{h} \sum_{i} I_{i}(h)\right]$, where $I_{i}$ is the $i$ th measurement and $\langle I(h)\rangle$ is the weighted mean of all measurements of $I(h)$. $\ddagger R_{\text {cryst }}=\sum_{h}\left|F_{\mathrm{o}}-F_{\mathrm{c}}\right| / \sum_{h} F_{\mathrm{o}}$, where $F_{\mathrm{o}}$ and $F_{\mathrm{c}}$ are the observed and calculated structure-factor amplitudes of reflection $h$. $\S R_{\text {free }}$ is $R_{\text {cryst }}$ for a randomly selected $5 \%$ of the data excluded from refinement (Brünger, 1992).

phate $\mathrm{pH} 9.0$ and incubated overnight at $310 \mathrm{~K}$ to catalyse deamidation of the N-terminal residue from Gln to pyroGlu (Blombäck, 1967) and then purified further by $\mathrm{C}_{4}$ reversedphase HPLC (Holloway et al., 2001). The purified material was finally lyophilized to dryness and reconstitued in AnalaRgrade water. Purity was $>95 \%$ as judged by SDS-PAGE, reversed-phase HPLC and electrospray ionization-mass spectrometry (data not shown). Protein concentration was determined from UV absorbance at $280 \mathrm{~nm}$ using an estimated $\varepsilon_{280}$ (Pace et al., 1995) of $6335 \mathrm{M}^{-1} \mathrm{~cm}^{-1}$.

\subsection{Crystallization and data collection}

Crystals were grown by the hanging-drop vapour-diffusion technique. Drops were prepared by mixing $2 \mu \mathrm{l}$ protein solution $\left(15 \mathrm{mg} \mathrm{ml}^{-1}\right)$ with $2 \mu \mathrm{l}$ reservoir solution and were incubated at $289 \mathrm{~K}$ suspended over $0.8 \mathrm{ml}$ reservoirs. Two types of crystal were grown, $\mathrm{mAng}-\mathrm{SO}_{4}$ and $\mathrm{mAng}-\mathrm{PO}_{4}$. In the former case the reservoir solution was $25 \%(w / v)$ PEG 4000, $0.2 \mathrm{M}$ $\left(\mathrm{NH}_{4}\right)_{2} \mathrm{SO}_{4}$ (unbuffered) and in the latter it was $25 \%(w / v)$ PEG 4000, $0.1 M$ sodium phosphate buffer $\mathrm{pH}$ 5.2. $\mathrm{mAng}_{-} \mathrm{SO}_{4}$ crystals were thin plates and reached their full size within $3 \mathrm{~d}$. mAng- $\mathrm{PO}_{4}$ crystals were thicker and grew more slowly, continuing their growth for 1-2 months.

Diffraction data were collected at room temperature on station PX9.6 of the Synchrotron Radiation Source (Daresbury, UK), which provided monochromatic radiation of $0.87 \AA$ 
wavelength and was equipped with an ADSC Quantum-4 $\mathrm{CCD}$ detector. Data from two $\mathrm{mAng}-\mathrm{SO}_{4}$ crystals were merged and supplemented with further data from an in-house MAR Research $30 \mathrm{~cm}$ image plate mounted on a Rigaku RU-H3R rotating-anode $\mathrm{X}$-ray source emitting $\mathrm{Cu} K \alpha$ radiation. Data from a single $\mathrm{mAng}-\mathrm{PO}_{4}$ crystal were collected at the synchrotron. All data were indexed, scaled and reduced using DENZO and SCALEPACK (Otwinowski \& Minor, 1997) and intensities were truncated to amplitudes using TRUNCATE (French \& Wilson, 1978). Detailed dataprocessing statistics are given in Table 1.

\subsection{Structure determination and refinement}

Initial phases were determined by the molecular-replacement method as implemented in AMoRe (Navaza, 1994), employing a search model derived from the crystal structure of bAng (PDB code 1agi; Acharya et al., 1995). In this search model, residues 1 and 121-125 were deleted and all nonglycine residues that differed from their counterparts in mAng were replaced by alanine. Clear solutions were obtained for both $\mathrm{mAng}-\mathrm{SO}_{4}$ and $\mathrm{mAng}-\mathrm{PO}_{4}$ and the resultant models were subjected to cycles of refinement in which simulated annealing, coordinate minimization and $B$-factor refinement with CNS (Brünger et al., 1998) were alternated with manual model building using $O$ (Jones et al., 1991). During the final stages, restrained refinement and electron-density map calculations were performed with REFMAC5 (Murshudov et al., 1997). Those residues with partial density were truncated accordingly and residues for which there was no discernible density were omitted. Polyvalent anions were then incorporated, followed by the first two shells of water molecules. All water molecules had peaks of $\geq 3 \sigma$ in $m F_{\mathrm{o}}-D F_{\mathrm{c}}$ electrondensity maps. Detailed statistics for each model are given in Table 1.

Model validation was conducted using PROCHECK (Laskowski et al., 1993) and the WHAT_CHECK server (Rodriguez et al., 1998). Structural superpositions were performed with LSQMAN (Kleywegt \& Jones, 1994) and topological classification with PROMOTIF3 (Hutchinson \& Thornton, 1996). Omit phases were calculated with SFCHECK (Vaguine et al., 1999). Figures were drawn using PyMOL (DeLano Scientific, San Carlos, CA, USA).

\section{Results}

\subsection{Structural overview}

$\mathrm{mAng}$ is a monomeric protein of 121 residues. It adopts the $\alpha / \beta$-fold characteristic of other angiogenins and members of

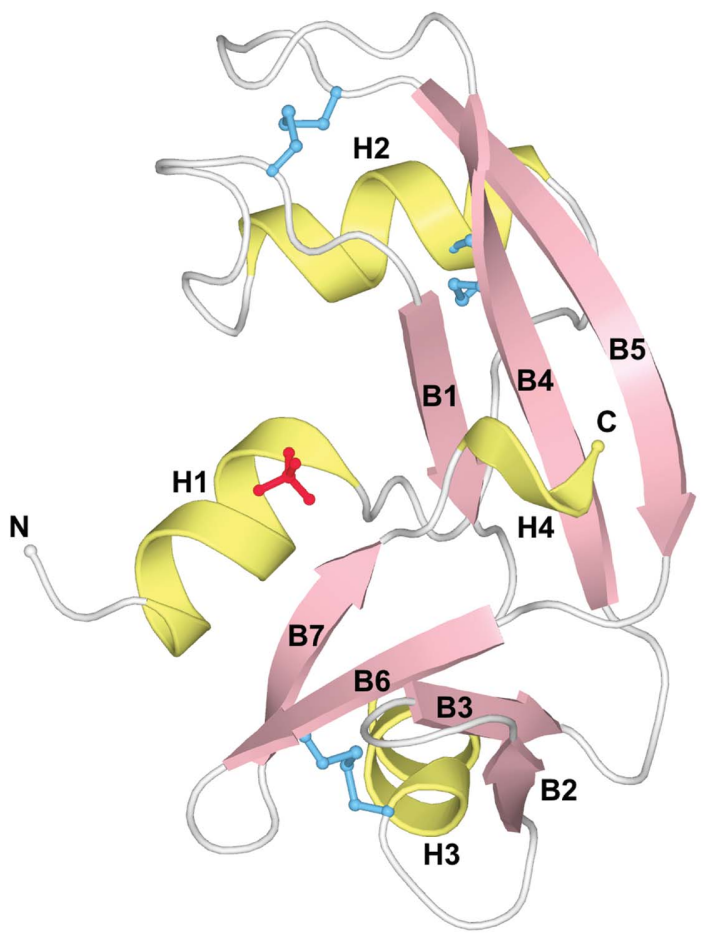

(a)

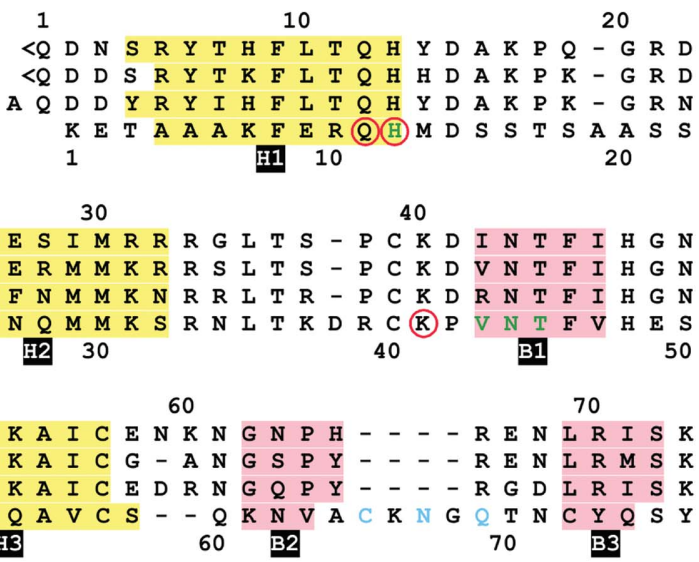

$80 \quad 90 \quad 100$

hAng

mAng

RNase A

mAng

bAng

RNase A

bAng

RNase A

$\mathrm{K}$ R

K S N I K A I C G A N S P Y - - R E I R M S K

K N D I K A I C E D R N G Q P Y - - R G D L R I S K

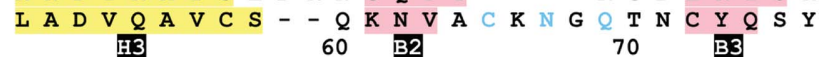

S S F Q V T T C K L H G G S P W P P C Q Y R A T A G F R

$S P F Q V T$ T C K H T G G S P R P P C Q Y R A S A G F R

S E F Q I T I C K H K G G S S R P P C R Y G A T E D S R

$S$ T MSITDCR E T G S S K Y P N C A Y K T T Q A N K

80 B4 90 100 B5

110

hAng N V V V A C E N - - G L P V H L D Q S I F R R P

mAng HV VIACE N - G L P V H F D E S F F S I

bAng VIVVGCE N- - G L P V H F D E F I T P R H

RNase A

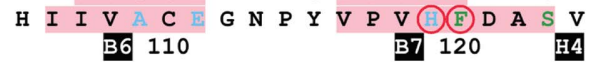

(b)

Figure 1

Structural overview. (a) Ribbon diagram of the mAng- $\mathrm{SO}_{4}$ structure. Helices are coloured yellow and $\beta$-strands pink. Disulfide bonds (blue) and a sulfate ion (red) bound to the active site are shown in ball-and-stick representation. Elements of secondary structure are labelled, as are the $\mathrm{N}$ - and $\mathrm{C}$ terminal extremities of the $\mathrm{C}^{\alpha}$ chain (residues 3 and 119, respectively). The disulfide bonds are formed between residues 39 and 91 (top), 26 and 80 (middle) and 57 and 106 (bottom). (b) Structure-based alignment of hAng, mAng, bAng and RNase A sequences. Elements of secondary structure are shaded according to the colour scheme in $(a)$ and labelled below. In the RNase $\mathrm{A}$ sequence, residues shown crystallographically to form the $\mathrm{B}_{1}$ and $\mathrm{B}_{2}$ subsites are coloured green and blue, respectively, while those that form the $\mathrm{P}_{1}$ subsite are ringed in red. hAng residue numbers are given above the sequences and RNase A residue numbers below. ' $<\mathrm{Q}$ ' signifies a pyroglutamate residue. 

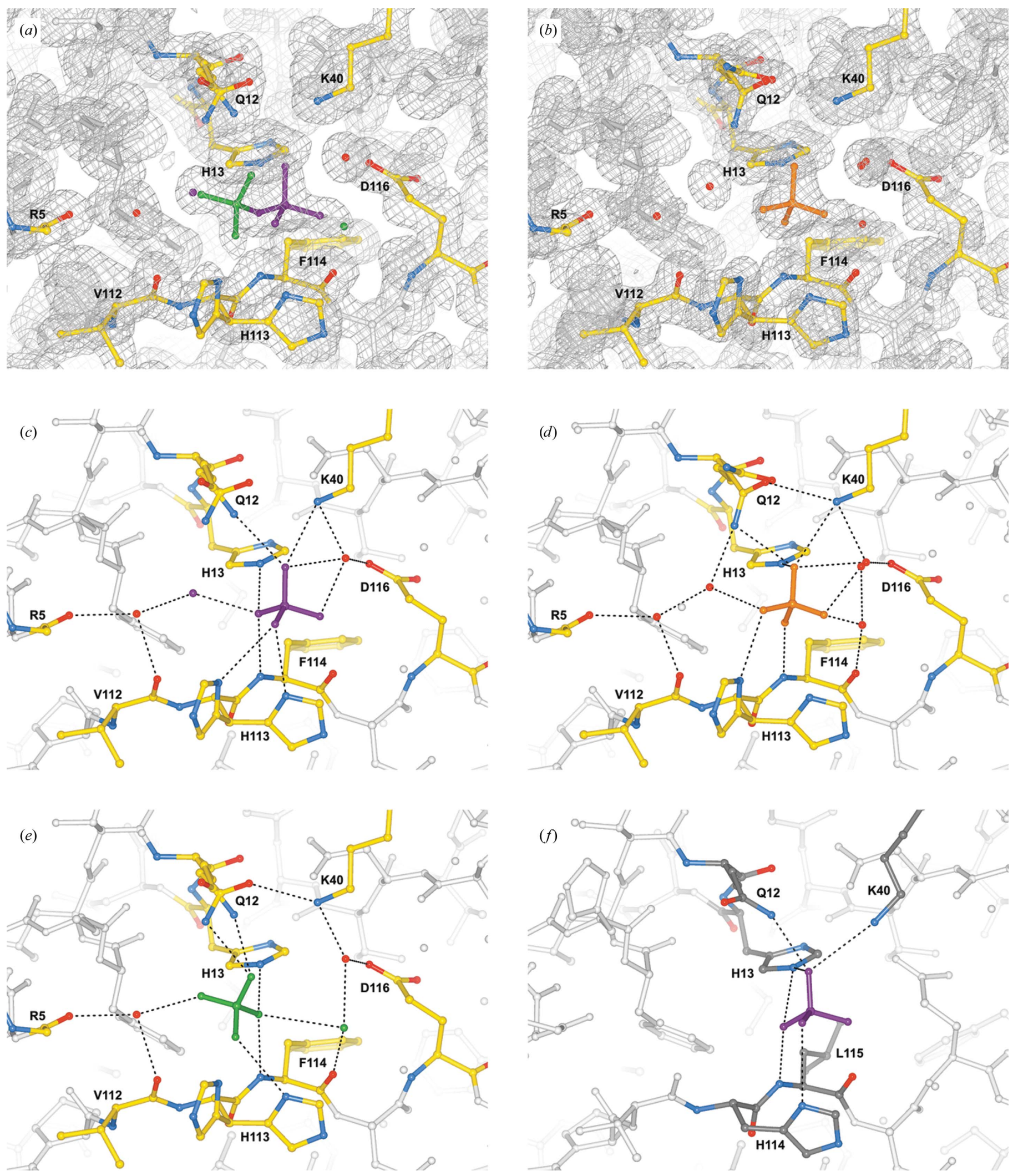

Figure 2

$\mathrm{P}_{1}$ subsite. Shown are $m F_{\mathrm{o}}$ OMIT electron-density maps contoured at $1 \sigma$ for $(a)$ the $\mathrm{mAng}-\mathrm{PO}_{4}$ and $(b)$ the $\mathrm{mAng}-\mathrm{SO}_{4}$ structures. Also shown are potential hydrogen bonds (dashed lines) in the $(c) \mathrm{mAng}-\mathrm{PO}_{4}$ (anion orientation $\left.A\right),(d) \mathrm{mAng}_{-} \mathrm{SO}_{4},(e) \mathrm{mAng}_{-} \mathrm{PO}_{4}($ anion orientation $B)$ and $(f)$ hAng- $\mathrm{PO}_{4}$ (PDB code 1hby; Leonidas et al., 2001) structures. Residues involved in anion coordination are labelled and highlighted with colours according to the following scheme: $\mathrm{mAng}$ carbon, gold; hAng carbon, grey; nitrogen, blue; oxygen, red. The $\mathrm{P}_{1}$ subsite of $\mathrm{mAn}_{\mathrm{A}}-\mathrm{PO}_{4}$ is occupied by phosphate ions in two orientations, $A$ (purple) and $B$ (green); adjacent orientation-specific water molecules are coloured likewise. Bound anions in the mAng- $\mathrm{SO}_{4}$ and hAng- $\mathrm{PO}_{4}$ structures are coloured orange and purple, respectively. The side chain of Arg5 is omitted for clarity. 
the pancreatic ribonuclease superfamily (Fig. 1a). When compared with hAng, it has two single-residue deletions, one at residues $59 / 60$ and one at the C-terminus (Fig. 1b). The former affects the numbering of residues 60-121, which have values of one less than their hAng counterparts.

The data enabled the modelling of the polypeptide backbone of residues 3-119 in the mAng- $\mathrm{SO}_{4}$ structure and residues 2-119 in the $\mathrm{mAng}-\mathrm{PO}_{4}$ structure. The topologies of the two structures are extremely similar, the $\mathrm{C}^{\alpha}$ atoms of residues 3-119 overlaying with an r.m.s. deviation of just $0.14 \AA$. They also superpose closely with the structures of hAng (PDB code 1b1i; Leonidas, Shapiro, Allen et al., 1999) and bAng (PDB code 1agi; Acharya et al., 1995): the $\mathrm{C}^{\alpha}$ atoms of $\mathrm{mAng}_{-} \mathrm{SO}_{4}$ (residues 4-58 and 60118) deviate from those of hAng (residues 4-58 and 61-119) and bAng (residues 5-59 and 62-120) by 0.62 and $0.66 \AA$ (r.m.s.), respectively; the corresponding figures for $\mathrm{mAng}-\mathrm{PO}_{4}$ are 0.60 and $0.64 \AA$, respectively. Excepting the $\mathrm{N}$ - and C-termini, the largest deviations between equivalent $\mathrm{C}^{\alpha}$ atoms of mAng and hAng occur in close proximity to glycine residues $20,48,58$ and 85 and are no larger than $1.9 \AA$. Of these residues, glycines 20, 48 and 85 are conserved and probably impart some flexibility to each structure. Gly58, however, is novel to $\mathrm{mAng}$ and precedes the single-residue deletion identified above. Structure-based alignment of mAng, hAng and bAng reveals a close correspondence of secondarystructure elements (Fig. 1b).

The electron-density maps for the $\mathrm{mAng}_{-} \mathrm{SO}_{4}$ and $\mathrm{mAng}-$ $\mathrm{PO}_{4}$ structures are of high quality (Figs. $2 a$ and $2 b$ ) and show that in each case the side chains of Gln12, Ser34, Ser51, Ser62, Met70, Ser96, His113 and Ser117 have two or more distinct conformations. Unique to the $\mathrm{mAng}-\mathrm{SO}_{4}$ model are dual conformations of Val42 and Ser51 and unique to the mAng$\mathrm{PO}_{4}$ model are dual conformations of Lys50, Lys72 and Ser73. The extremities of Asp3, Arg5, Lys8, Lys17, Lys19, Arg24, Arg28, Lys31, Arg32, Ser37, Asp41, Asn67, Lys81, Ser86, Arg88, Gln92, Arg94, Glu107 and Ser120 show more significant disorder and cannot be modelled.

\subsection{Structure of the $P_{1}$ subsite}

Residues of the $\mathrm{P}_{1}$ subsite bind and catalyse the cleavage of the scissile phosphodiester linkage of RNA substrates. At this subsite in RNase A, the side chains of His12, Lys41 and His119 are directly involved in catalysis, while the side chain of Gln11 and the main chain of Phe120 make significant hydrogen bonds with the phosphodiester moiety (Raines, 1998 and references therein). In mAng, the catalytic triad is conserved in the form of His13, Lys40 and His113. The positions and conformations of these residues are highly similar in each of the two mAng structures and are in accordance with their likely catalytic function. The side chain of His113 is observed in two conformations that correspond to those designated $A$ $\left(\chi_{1} \simeq 160^{\circ}, \chi_{2} \simeq 80^{\circ}\right)$ and $B\left(\chi_{1} \simeq-75^{\circ}, \chi_{2} \simeq-55^{\circ}\right)$ in RNase A (Borkakoti et al., 1982). In the $\mathrm{mAng}-\mathrm{PO}_{4}$ structure, conformation $A$ has greater occupancy $(\sim 80 \%)$, while in the mAng- $\mathrm{SO}_{4}$ structure the two conformations are evenly occupied. Each structure also reveals that an anion from the crystallization medium is ligated to the catalytic triad, the side chain of Gln12 (the counterpart of RNase A Gln11) and the main-chain $\mathrm{N}$ atom of Phe114 (the counterpart of RNase A Phe120) (Fig. 2, Table 2). Two conformations of the Gln12 side chain are present in each structure, designated $A$ and $B$. The most significant difference between conformations $A$ and $B$ is an $\sim 100^{\circ}$ rotation of the terminal amide group.

There are minor differences between mAng and hAng in the conformations of some of the $\mathrm{P}_{1}$ subsite residues. In crystal structures of hAng, His114 (the counterpart of mAng His113) has been observed in conformation $A$ only, although a dynamic equilibrium between the $A$ and $B$ conformers has been deduced from NMR spectroscopy of hAng solutions (Lequin et al., 1997). The conformations of Lys40 in the mAng and hAng (free or phosphate-bound) structures differ such that their $\mathrm{N}^{\zeta}$ atoms are displaced by $\simeq 1.5 \AA$, while the orientations of the Gln12 side-chain amide group also differ. These differences are difficult to explain in the absence of the structure of unliganded mAng, but they may reflect fine differences in anion binding, described below.

Although there are obvious similarities between phosphate and sulfate ions, there are differences in the way that each binds to the $\mathrm{P}_{1}$ subsite of $\mathrm{mAng}$. Phosphate binds in two equally populated but markedly different orientations that have a common position for one $\mathrm{O}$ atom, which we designate O1. In orientation $A$, the anion is particularly well coordinated, making hydrogen bonds to five $\mathrm{P}_{1}$-subsite residues: Gln12 (conformation A), His13, Lys40, His113 (both conformations) and Phe115 (Fig. 2c, Table 2). This represents all 
possible interactions contained within the crystalline complexes of RNase A with phosphate (PDB code 5rsa; Wlodawer \& Sjölin, 1983), sulfate (e.g. PDB codes 3rn3 and 1dy5; Howlin et al., 1989; Esposito et al., 2000) or dinucleotide inhibitors (e.g. PDB code 1rpg; Zegers et al., 1994). Orientation $A$ is also similar to the single orientation observed in the hAng- $\mathrm{PO}_{4}$ structure (PDB code 1hby; Leonidas et al., 2001; Fig. 2f), but with somewhat better hydrogen-bonding geometry (Table 2). Orientation $B$ is substantially different and to our knowledge is novel (Fig. 2e). It is incompatible with conformation $B$ of His113, but permits hydrogen bonds with all the above $\mathrm{P}_{1}$ residues except Lys40. Sulfate binds to the $\mathrm{P}_{1}$ subsite in a single orientation that is offset slightly from phosphate orientation $A$ (Fig. $2 d$ ). Although some specifics of the hydrogen bonds are different (His13 bonds to $\mathrm{O} 3$ instead of $\mathrm{O} 1$, His113 bonds in conformation $A$ only and Gln12 bonds in conformation $A$ rather than conformation $B$; Table 2 ), the same five residues ligate the anion.

\subsection{Structure of the $B_{1}$ subsite and $C$ - terminal segment}

The $\mathrm{B}_{1}$ subsite binds the nucleotide base positioned upstream of the scissile phosphodiester bond and has been well characterized in RNase A (and derivatives thereof), where it takes the form of a narrow pocket comprising residues His12, Val43, Asn44, Thr45, Phe120 and Ser123. The primary component of the site is Thr45, which forms hydrogen bonds with the polar groups at positions 2 and 3 of pyrimidine bases (Richards et al., 1970; Wlodawer et al., 1983; delCardayré \& Raines, 1995). Mutational analyses indicate that hAng Thr44 is the functional counterpart of RNase Thr45 (Curran, Shapiro \& Riordan, 1993; Holloway et al., 2004). Crystal structures of wild-type proteins show that hAng residues His13, Ile42, Asn43 and Leu115 are the spatial counterparts of RNase A residues His12, Val43, Asn44 and Phe120 (Acharya et al., 1994; Leonidas, Shapiro, Allen et al., 1999). However, the anticipated binding position of the pyrimidine is obstructed by Gln117 and Phe120 and the only reasonable way in which hAng can bind RNA substrates requires a major structural rearrangement of the C-terminal region (residues 117-123; Leonidas et al., 2002).

The residues comprising the likely $\mathrm{B}_{1}$ subsites of $\mathrm{mAng}$ and hAng are partially conserved. There are two amino-acid substitutions: in mAng, Val42 replaces Ile42 and Phe114 replaces Leu115. Nevertheless, the $B_{1}$-subsite residues of mAng occupy positions similar to their hAng counterparts and are likely to play similar roles (Fig. 3). The site is obstructed by Glu116, the side chain of which makes two hydrogen bonds with Thr44 that are analogous to those made between Gln117 and Thr44 in hAng (Table 3). The side chain of Thr44 is also hydrogen bonded to the side chain of Thr79, replicating the Thr44-Thr80 interaction found in hAng. The other potential obstructor of the $\mathrm{B}_{1}$ subsite, Phe119, assumes a less invasive position than its hAng counterpart (Phe120; Fig. 3). Superposition with the RNase A-uridine vanadate complex (PDB code 1ruv; Ladner et al., 1997) indicates that the side chain of Phe119 (atom $\mathrm{C}^{\delta 2}$ ) is $3.6 \AA$ distant from the heteroatom at position 4 of the pyrimidine, somewhat further away than its hAng counterpart is (atom $\mathrm{C}^{\beta}$ at $2.3 \AA$ ). The side chains of these corresponding Phe residues also differ noticeably in conformation, their $\chi_{2}$ torsion angles differing by $37-39^{\circ}$. These differences appear to be a consequence of the $\mathrm{Leu} \rightarrow$ Phe substitution at mAng position 114. Indeed, Phe114 
and Phe119 make direct contact, leading to a slight reorientation of Phe119 and downstream residues of the C-terminal segment that raises the $\mathrm{mAng}$ versus $\mathrm{hAng} \mathrm{C}^{\alpha}$ deviation from $0.3 \AA$ at position 117 to $2.3 \AA$ at position 119 (mAng numbering). This is also a characteristic of the bAng structure, which features an equivalent $\mathrm{Leu} \rightarrow$ Phe substitution.

The closed conformation of hAng is stabilized by Gln117Thr44 and Ser118-Asp116 hydrogen bonds and by the hydrophobic packing of the Ile119 and Phe120 side chains against the main body of the protein (Harper \& Vallee, 1988; Curran, Shapiro, Riordan et al., 1993; Russo et al., 1994, 1996; Shapiro, 1998; Leonidas et al., 2002). In mAng, the hydrogen bonds are comparable (Table 3) while, as a consequence of several amino-acid substitutions (Phe114 for Leu115, Phe118 for Ile119, Tyr64 for His65), the hydrophobic interactions are more extensive. In more detail, the side chains of Phe118 and

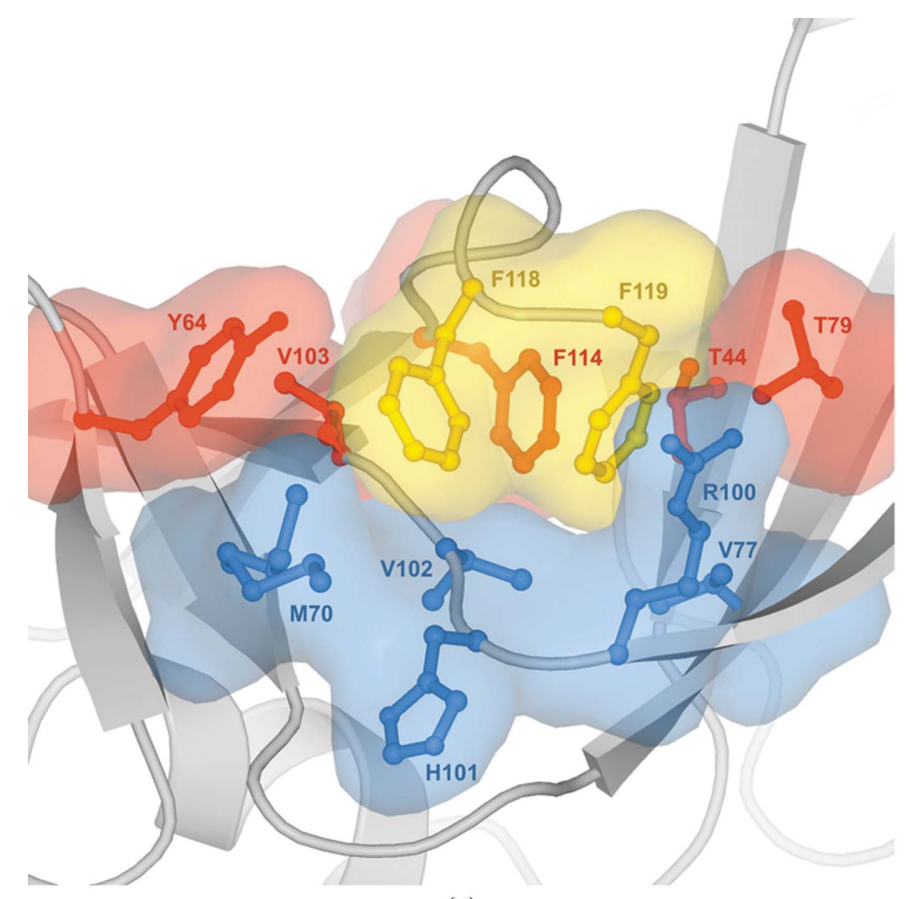

(a)

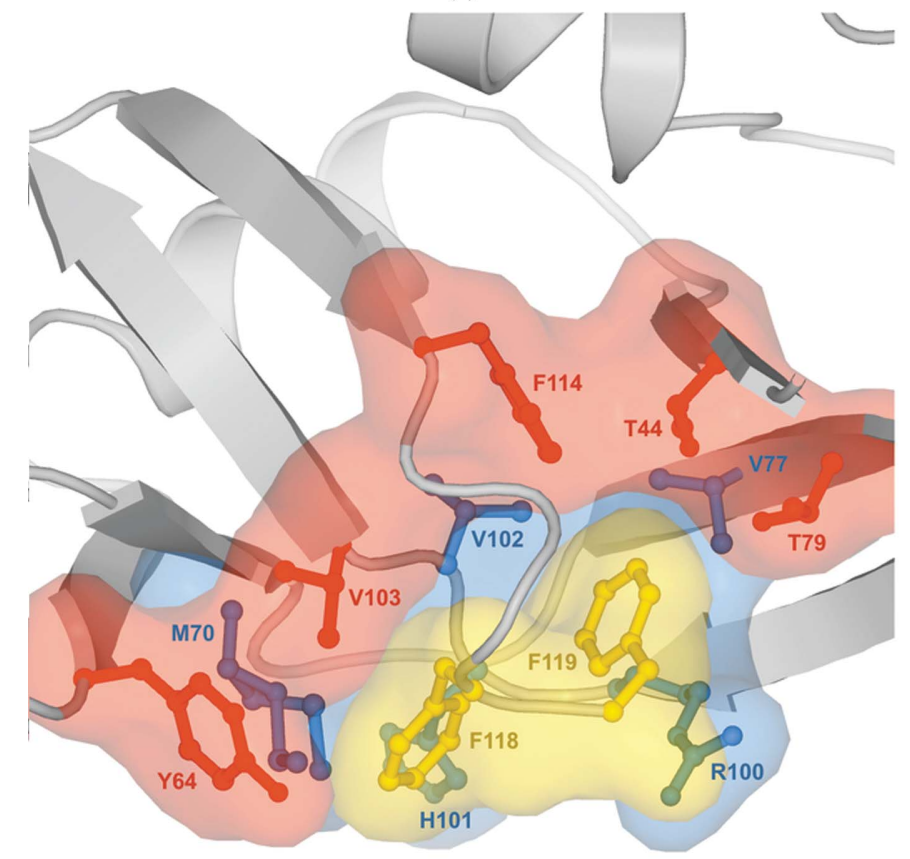

(c)

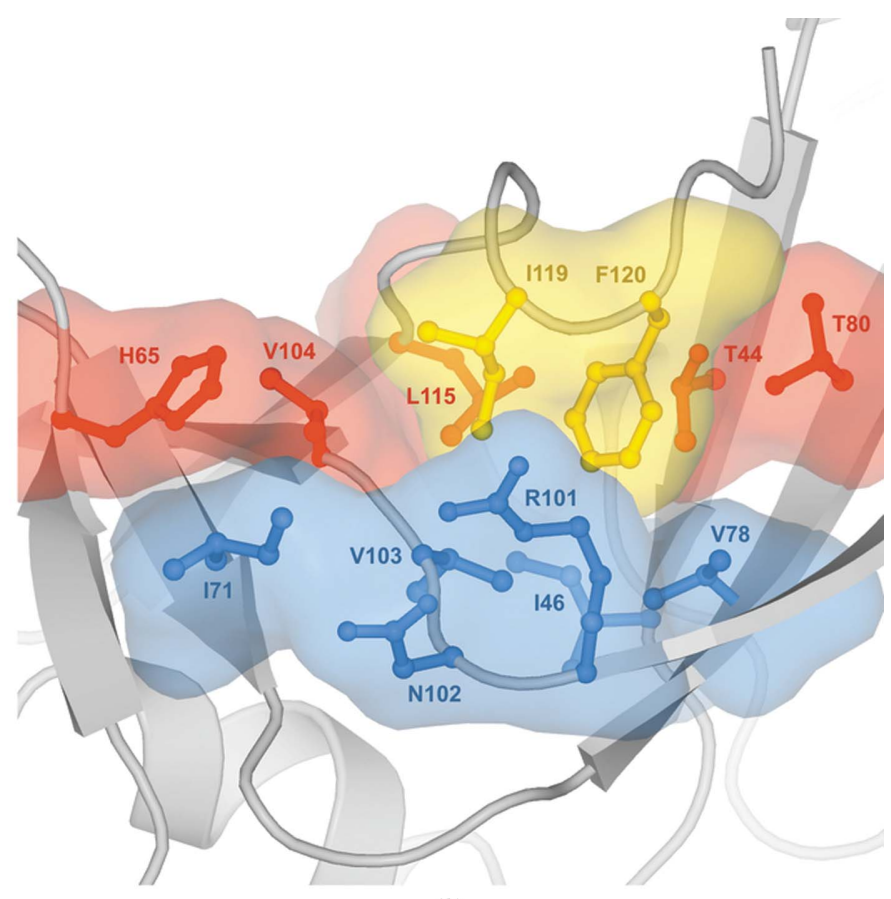

(b)

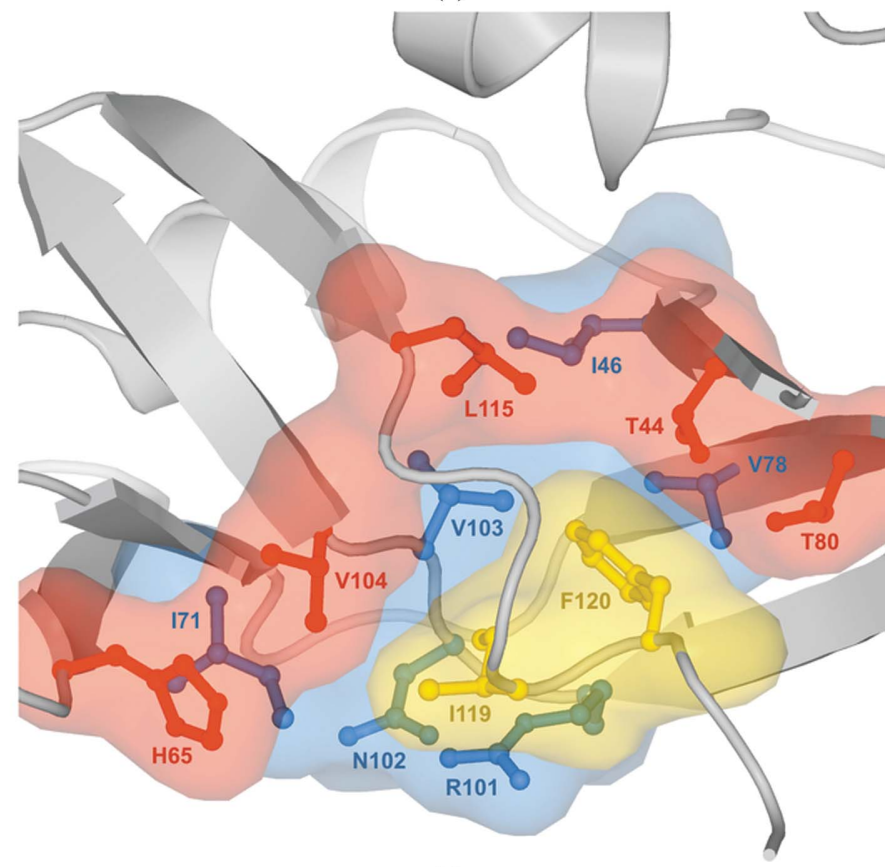

(d)

Figure 4

Hydrophobic packing of the C-terminus. $(a)$ and $(c), \mathrm{mAng}_{-} \mathrm{SO}_{4} .(b)$ and $(d)$, hAng (PDB code 1b1i; Leonidas, Shapiro, Allen et al., 1999). Hybrid representation in which secondary structures (grey) are shown in schematic form and side chains of C-terminal hydrophobic residues (gold), the pocket base (blue) and collar (red) are shown in both ball-and-stick and opaque space-filling forms. mAng Met70 is modelled in dual conformation. $(c)$ and $(d)$ were obtained from $(a)$ and $(b)$, respectively, by a $90^{\circ}$ rotation about the $x$ axis. 
Phe119 (the counterparts of Ile119 and Phe120 in hAng) are cradled in a pocket lined by residues from six $\beta$-strands (Fig. 4). These residues can be divided into those that form the pocket base (Met70, Val77, Arg100, His101 and Val102) and those that form a collar (Thr44, Tyr64, Thr79, Val103 and Phe114). Both Phe118 and Phe119 make extensive contacts with residues from each layer (Figs. $4 a$ and $4 c$ ). In contrast, Ile119 and Phe120 of hAng interact closely with the base (and with each other) but much less so with the collar (Figs. $4 b$ and $4 d$ ).

\subsection{Structure of the $B_{2}$ subsite and the H3-B2 loop}

The $\mathrm{B}_{2}$ subsite binds the nucleotide base positioned downstream of the scissile phosphodiester bond. In RNase A, adenine binding involves nonpolar interactions with the side chains of His119 ( $\pi-\pi$ stacking), Cys65 and Ala109, strong hydrogen bonds to the side chain of Asn71 and transient hydrogen bonds to Asn67, Gln69 and Glu111 (Richards et al., 1970; Tarragona-Fiol et al., 1993; Fontecilla-Camps et al., 1994; Zegers et al., 1994; Toiron et al., 1996). Several of these residues are contributed by a disulfide-stabilized loop (residues 65-72) located between strands B2 and B3. In Ang-family members, this loop and its disulfide bridge are not conserved and the structure of the $\mathrm{B}_{2}$ subsite is not easily discerned (Leonidas, Shapiro, Allen et al., 1999). The subsite may be rather rudimentary, perhaps limited to counterparts of the nonpolar elements of the RNase A subsite, i.e. residues His114, Leu69 and Ala106 in hAng. These three residues are conserved in mAng and occupy positions similar to their hAng counterparts. Any interactions that they make with substrates are likely to be conserved.

The roles of RNase A and Ang residues in the region of strand B2 have diverged over the course of evolution. Whereas several of these residues are employed by RNase A for substrate binding (as described above), a subset (residues 60-68 in hAng) is implicated in the interaction of Ang with the surface of endothelial cells (Leonidas, Shapiro, Allen et al., 1999). Among these is Asn61, which is located in the solventexposed loop that connects strand B2 to the preceding helix, H3. Proteolytic cleavage of the 60/61 peptide bond or deamidation of Asn61 to isoAsp abolishes angiogenic activity, but has no appreciable effect on enzymatic activity (Hallahan et al., 1991, 1992). Variants with either of these modifications lack the ability to inhibit Ang-induced neovascularization, indicating the perturbation of a critical cell-binding site. The importance of Asn61 may lie in its ability to interact directly with cell-surface molecules or in its role in stabilizing the structure of two adjacent regions with which it forms three hydrogen bonds (Asn61 N ${ }^{\delta 2}-$ Ser52 O, Asn61 $\mathrm{N}^{\delta 2}-$ Ser52 $\mathrm{O}^{\gamma}$ and Ser74 $\mathrm{N}-\mathrm{Asn} 61 \mathrm{O}^{\delta 1}$ ). The structure of $\mathrm{mAng}$ narrows down these possibilities. Whereas in hAng the H3-B2 connection is a type I $\beta$-turn comprising four residues (Glu58Asn59-Lys60-Asn61), in mAng the connection comprises only three (Gly58-Ala59-Asn60) and features two nonconservative substitutions. The electron-density map for this region is well defined in the $\mathrm{mAng}-\mathrm{SO}_{4}$ and $\mathrm{mAng}-\mathrm{PO}_{4}$ structures and shows that the type I $\beta$-turn is present in a modified form (Gly58-Ala59-Asn60-Gly61). The extended torsional freedom of the two glycine residues in the turn prevents perturbation of helix H3 and strand B2. Asn60 (the counterpart of hAng Asn61) makes no direct contact with the rest of the protein (Fig. 5). Instead, the Asn60 side chain adopts a more solventexposed orientation in which it is free to hydrogen bond with Lys72 from a symmetry-related molecule (not shown). A network of water molecules separates Asn60 from Ser52 and Asn73 (the counterparts of hAng Ser52 and Ser74), suggesting that the side chain of Asn 60/61 is not essential for the positioning of residues 52 and 73/74.

\subsection{Crystal packing and the potential binding of inhibitors}

Crystal packing orients the central substrate-binding subsites of $\mathrm{mAng}\left(\mathrm{B}_{1}, \mathrm{P}_{1}\right.$ and $\left.\mathrm{B}_{2}\right)$ towards a well defined solvent channel. The accessibility of these subsites to a known dinucleotide inhibitor such as $5^{\prime}$-phospho-2'-deoxyuridine-3'pyrophosphate $\left(\mathrm{P}^{\prime} \rightarrow 5^{\prime}\right)$ adenosine $3^{\prime}$-phosphate (pdUppA$3^{\prime}$-p; Russo et al., 2001) can be gauged by aligning each of the mAng structures with that of the RNase A-pdUppA-3'-p complex (PDB code 1qhc; Leonidas, Shapiro, Irons et al., 1999) and superposing the dinucleotide coordinates with those of mAng. This type of analysis suggests that the pathway of such a ligand to the active site (the obstructive conformation of the C-terminus aside) is clear (Fig. 6a). Furthermore, none of the likely dinucleotide-binding residues make intermolecular crystal contacts.

In order for a given small-molecule inhibitor to bind to Ang, it may be necessary for the $\mathrm{C}$-terminus to disengage from the main body of the protein. For this to occur in a crystal-soaking experiment, there will need to be sufficient room for manoeuvre of the C-terminus within the crystal. In mAng crystals, the C-terminal segment approaches two symmetryrelated molecules (Fig. $6 b$ ), which we designate SYM MOL 1 (lattice position $-b+c$, symmetry operator $-x+\frac{1}{2}, y+\frac{1}{2},-z$ ) and SYM MOL 2 (lattice position $-b$, symmetry operator $\left.-x+\frac{1}{2}, y+\frac{1}{2},-z\right)$. Owing to different degrees of ordering at their N-termini, the $\mathrm{mAng}-\mathrm{SO}_{4}$ and $\mathrm{mAng}-\mathrm{PO}_{4}$ structures differ in the extent of the interaction. In $\mathrm{mAng}-\mathrm{PO}_{4}$ (which has the best ordered $\mathrm{N}$-terminus), the $\mathrm{C}^{\varepsilon 1}$ atom of Phe118 lies $3.7 \AA$ from the $\mathrm{C}^{\beta}$ atom of Asp2 from SYM MOL 1. The two side chains make contact 'end-on' and the C-terminal segment does not appear to be constrained by the interaction. Furthermore, in $\mathrm{mAng}-\mathrm{SO}_{4}$ the side chains of all residues upstream of Ser 4 are disordered and no contact is evident. The C-terminal segment also approaches helix $\mathrm{H} 2$ and the preceding loop of SYM MOL 2 but does not make direct contact (Fig. 6b). Here, the main-chain conformation orientates the side chains of Lys19, Arg24 and Arg28 toward the $\mathrm{C}$-terminal segment. However, in the $\mathrm{mAng}-\mathrm{PO}_{4}$ structure none of these side chains are ordered beyond $\mathrm{C}^{\beta}$. In the mAng- $\mathrm{SO}_{4}$ structure Arg24 shows electron density out to $\mathrm{C}^{\delta}$, but the other two are again disordered beyond $\mathrm{C}^{\beta}$. Thus, it appears that these side chains have considerable mobility within either crystal form. 
In summary, mAng crystals appear to offer small-molecule inhibitors ample access to the active site and may even withstand a modest change in the conformation of the C-terminal segment.

\section{Discussion}

As might be expected on consideration of sequence homology alone, the structure of mAng is extremely similar to that of hAng. Nevertheless, minor differences between the two orthologues have provided some insight into RNA-cleavage activity and cell binding. There are subtle differences between the enzymatic properties of mAng and hAng (Nobile et al., 1996; Shapiro, 1998). Firstly, the specific activity of mAng is only about one-third of that of hAng as judged by the ability to cleave tRNA or dinucleotide substrates. This seems most likely to be a consequence of enhanced hydrophobic interactions at the C-terminus of mAng which stabilize the 'closed' conformation, presenting a higher energetic barrier to substrate turnover. This view is supported by studies on the contribution of hydrophobic interactions to the C-terminal conformation of hAng. When these interactions are weakened through the introduction of mutations at the C-terminus (I119A/F120A) or by exposure to methanol, enzymatic activity is enhanced threefold to fourfold but substrate specificity is unaffected (Russo et al., 1996; Leonidas et al., 2002). Hence, an increase in the hydrophobic interactions as observed in mAng would be likely to cause a severalfold suppression of enzymatic activity. Next, whereas hAng has a 12-fold preference for $\mathrm{C}$ over $\mathrm{U}$ at the $\mathrm{B}_{1}$ subsite, $\mathrm{mAng}$ has a somewhat stronger 35 -fold preference. This suggests modification of the recognition of the substituent(s) at positions 3 and/or 4 of the pyrimidine. There are no structural differences in the vicinity of Thr44 that might affect its ability to form hydrogen bonds with the nitrogen function at position 3 and the nature of the interaction with the heteroatom at position 4 is unknown. Nearby, two nonpolar residues are conservatively substituted (hAng Ile42 $\rightarrow$ mAng Val42, hAng Leu115 $\rightarrow$ mAng Phe114) and there is a small shift in the alignment of the C-terminal segment. However, it is not possible to predict what bearing this might have on $\mathrm{C} / \mathrm{U}$ selectivity and the resolution of this issue awaits a direct observation of pyrimidine binding. In contrast, $\mathrm{mAng}$ and $\mathrm{hAng}$ have very similar selectivities favouring $A$ over $G$ at the $B_{2}$ subsite (fourfold and threefold, respec-

Figure 5

$\mathrm{B} 2-\mathrm{H} 3$ loop. Stereoview in which $\mathrm{mAng}-\mathrm{SO}_{4}$ (carbon, gold; nitrogen, blue; oxygen, red; sulfur, orange) is superposed with hAng (PDB code 1b1i; Leonidas, Shapiro, Allen et al., 1999; grey). Residues that interact with mAng Asn60 and hAng Asn61 are also shown. Dashed lines denote potential hydrogen bonds.

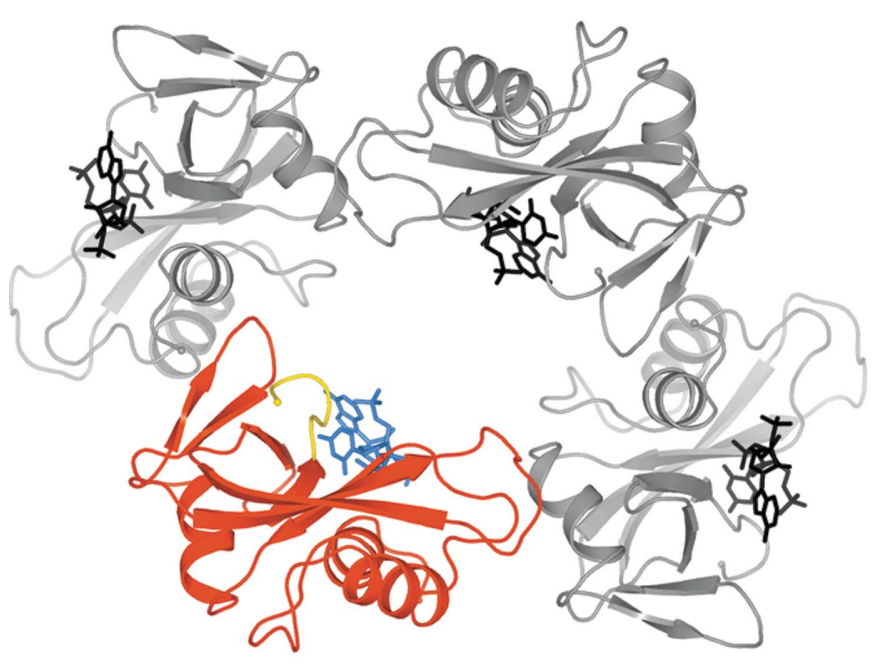

(a)

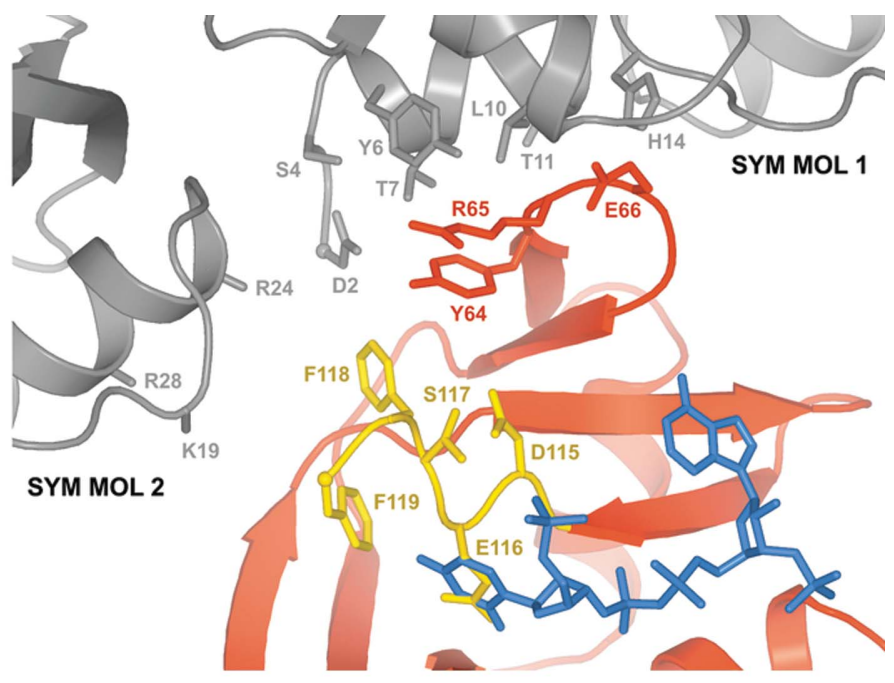

(b)

Figure 6

Packing of mAng- $\mathrm{PO}_{4}$ crystals. (a) Transverse section through a solvent channel. (b) Details of the interactions between the C-terminus and symmetryrelated neighbours SYM MOL 1 and SYM MOL 2 (see text for details). Shown are mAng (residues 2-114 in red, residues 115-119 in gold), a superposed pdUppA-3'-p molecule obtained by alignment of the mAng- $\mathrm{PO}_{4}$ monomer with the RNase A-pdUppA-3'-p complex (PDB code 1qhe; Leonidas, Shapiro, Irons et al., 1999; blue) and selected symmetry-related neighbours (grey/black). The side chains of Lys19, Arg24 and Arg28 are disordered beyond $\mathrm{C}^{\beta}$. 
tively). This is consistent with the conservation of the identity and positioning of the putative $\mathrm{B}_{2}$ subsite residues His114, Leu69 and Ala106 (hAng numbering). Finally, the mAng structure supports the view that the side chain of Asn61 in hAng binds directly to a molecule on the endothelial cell surface and does not merely stabilize adjacent segments of the hAng structure.

The structures presented here indicate that in several respects crystalline mAng is a promising tool for the study of Ang structure-function relationships, particularly those relating to enzymatic activity. Firstly, the catalytic and specificity-determining residues of the two orthologues superpose closely, suggesting that small-molecule inhibitors targeted to the central substrate-binding subsites will bind to each orthologue in similar ways. Secondly, the crystal packing arrangement should permit the approach of such inhibitors to their targets and there is some (albeit limited) scope for conformational rearrangement of the $\mathrm{C}$-terminus that might provide access to the cryptic $B_{1}$ subsite. These features represent considerable advantages over crystalline hAng and bAng.

The authors would like to thank the staff at the Synchrotron Radiation Source (Daresbury, UK) for their help during X-ray data collection and Dr Robert Shapiro for constructive criticism of the manuscript. This work was supported by the Wellcome Trust (UK) Programme Grant 067288 to KRA.

\section{References}

Acharya, K. R., Shapiro, R., Allen, S. C., Riordan, J. F. \& Vallee, B. L. (1994). Proc. Natl Acad. Sci. USA, 91, 2915-2919.

Acharya, K. R., Shapiro, R., Riordan, J. F. \& Vallee, B. L. (1995). Proc. Natl Acad. Sci. USA, 92, 2949-2953.

Blombäck, B. (1967). Methods Enzymol. 11, 398-411.

Bond, M. D. \& Vallee, B. L. (1990). Biochem. Biophys. Res. Commun. 171, 988-995.

Borkakoti, N., Moss, D. M. \& Palmer, R. A. (1982). Acta Cryst. B38, 2210-2217.

Brown, W. E., Nobile, V., Subramanian, V. \& Shapiro, R. (1995). Genomics, 29, 200-206.

Brünger, A. T. (1992). Nature (London), 355, 472-474.

Brünger, A. T., Adams, P. D., Clore, G. M., DeLano, W. L., Gros, P., Grosse-Kunstleve, R. W., Jiang, J.-S., Kuszewski, J., Nilges, M., Pannu, N. S., Read, R. J., Rice, L. M., Simonson, T. \& Warren, G. L. (1998). Acta Cryst. D54, 905-921.

Cho, S., Beintema, J. J. \& Zhang, J. (2005). Genomics, 85, 208-220.

Curran, T. P., Shapiro, R. \& Riordan, J. F. (1993). Biochemistry, 32, 2307-2313.

Curran, T. P., Shapiro, R., Riordan, J. F. \& Vallee, B. L. (1993). Biochim. Biophys. Acta, 1202, 281-286.

delCardayré, S. B. \& Raines, R. T. (1995). J. Mol. Biol. 252, 328-336.

Esposito, L., Vitagliano, L., Sica, F., Sorrentino, G., Zagari, A. \& Mazzarella, L. (2000). J. Mol. Biol. 297, 713-732.

Fett, J. W., Strydom, D. J., Lobb, R. R., Alderman, E. M., Bethune, J. L., Riordan, J. F. \& Vallee, B. L. (1985). Biochemistry, 24, 54805486.

Fontecilla-Camps, J. C., de Llorens, R., le Du, M. H. \& Cuchillo, C. M. (1994). J. Biol. Chem. 269, 21526-21531.

French, S. \& Wilson, K. (1978). Acta Cryst. A34, 517-525.

Fu, X. \& Kamps, M. P. (1997). Mol. Cell. Biol. 17, 1503-1512.
Fu, X., Roberts, W. G., Nobile, V., Shapiro, R. \& Kamps, M. P. (1999). Growth Factors, 17, 125-137.

Hallahan, T. W., Shapiro, R., Strydom, D. J. \& Vallee, B. L. (1992). Biochemistry, 31, 8022-8029.

Hallahan, T. W., Shapiro, R. \& Vallee, B. L. (1991). Proc. Natl Acad. Sci. USA, 88, 2222-2226.

Harper, J. W. \& Vallee, B. L. (1988). Proc. Natl Acad. Sci. USA, 85, 7139-7143.

Harper, J. W. \& Vallee, B. L. (1989). Biochemistry, 28, 1875-1884.

Holloway, D. E., Chavali, G. B., Hares, M. C., Baker, M. D., Subbarao, G. V., Shapiro, R. \& Acharya, K. R. (2004). Biochemistry, 43, 12301241.

Holloway, D. E., Hares, M. C., Shapiro, R., Subramanian, V. \& Acharya, K. R. (2001). Protein Expr. Purif. 22, 307-317.

Holloway, D. E., Shapiro, R., Hares, M. C., Leonidas, D. D. \& Acharya, K. R. (2002). Biochemistry, 41, 10482-10489.

Howlin, B., Moss, D. S. \& Harris, G. W. (1989). Acta Cryst. A45, 851861.

Hutchinson, E. G. \& Thornton, J. M. (1996). Protein Sci. 5, 212-220.

Jones, T. A., Zou, J. Y., Cowan, S. W. \& Kjeldgaard, M. (1991). Acta Cryst. A47, 110-119.

Kao, R. Y. T., Jenkins, J. L., Olson, K. A., Key, M. E., Fett, J. W. \& Shapiro, R. (2002). Proc. Natl Acad. Sci. USA, 99, 10066-10071.

Kishimoto, K., Liu, S., Tsuji, T., Olson, K. A. \& Hu, G.-F. (2005). Oncogene, 24, 445-456.

Kleywegt, G. J. \& Jones, T. A. (1994). Jnt CCP4/ESF-EACBM Newsl. Protein Crystallogr. 31, 9-14.

Kurachi, K., Davie, E. W., Strydom, D. J., Riordan, J. F. \& Vallee, B. L. (1985). Biochemistry, 24, 5494-5499.

Ladner, J. E., Wladkowski, B. D., Svensson, L. A., Sjölin, L. \& Gilliland, G. L. (1997). Acta Cryst. D53, 290-301.

Laskowski, R. A., MacArthur, M. W., Moss, D. S. \& Thornton, J. M. (1993). J. Appl. Cryst. 26, 283-291.

Leonidas, D. D., Chavali, G. B., Jardine, A. M., Li, S., Shapiro, R. \& Acharya, K. R. (2001). Protein Sci. 10, 1669-1676.

Leonidas, D. D., Shapiro, R., Allen, S. C., Subbarao, G. V., Veluraja, K. \& Acharya, K. R. (1999). J. Mol. Biol. 285, 1209-1233.

Leonidas, D. D., Shapiro, R., Irons, L. I., Russo, N. \& Acharya, K. R. (1999). Biochemistry, 32, 10287-10297.

Leonidas, D. D., Shapiro, R., Subbarao, G. V., Russo, A. \& Acharya, K. R. (2002). Biochemistry, 41, 2552-2562.

Lequin, O., Thuring, H., Robin, M. \& Lallemand, J. Y. (1997). Eur. J. Biochem. 250, 712-726.

McDonald, I. K. \& Thornton, J. M. (1994). J. Mol. Biol. 238, 777-793.

Moroianu, J. \& Riordan, J. F. (1994). Biochem. Biophys. Res. Commun. 203, 1765-1772.

Murshudov, G. N., Vagin, A. A. \& Dodson, E. J. (1997). Acta Cryst. D53, 240-255.

Navaza, J. (1994). Acta Cryst. A50, 157-163.

Nobile, V., Vallee, B. L. \& Shapiro, R. (1996). Proc. Natl Acad. Sci. USA, 93, 4331-4335.

Olson, K. A., Byers, H. R., Key, M. E. \& Fett, J. W. (2001). Clin. Cancer Res. 7, 3598-3605.

Olson, K. A., Byers, H. R., Key, M. E. \& Fett, J. W. (2002). Int. J. Cancer, 98, 923-929.

Olson, K. A., Fett, J. W., French, T. C., Key, M. E. \& Vallee, B. L. (1995). Proc. Natl Acad. Sci. USA, 92, 442-446.

Otwinowski, Z. \& Minor, W. (1997). Methods Enzymol. 276, 307-326.

Pace, C. N., Vajdos, F., Fee, L., Grimsley, G. \& Gray, T. (1995). Protein Sci. 4, 2411-2423.

Raines, R. T. (1998). Chem. Rev. 98, 1045-1065.

Richards, F. M., Wyckoff, H. W. \& Allewell, N. (1970). In The Neurosciences: Second Study Program, edited by F. O. Schmitt, pp. 901-912. New York: Rockefeller University Press.

Rodriguez, R., Chinea, G., Lopez, N., Pons, T. \& Vriend, G. (1998). Bioinformatics, 14, 523-528.

Russo, A., Acharya, K. R. \& Shapiro, R. (2001). Methods Enzymol. 341, 629-648. 
Russo, N., Nobile, V., Di Donato, A., Riordan, J. F. \& Vallee, B. L. (1996). Proc. Natl Acad. Sci. USA, 93, 3243-3247.

Russo, N., Shapiro, R., Acharya, K. R., Riordan, J. F. \& Vallee, B. L. (1994). Proc. Natl Acad. Sci. USA, 91, 2920-2924.

Shapiro, R. (1998). Biochemistry, 37, 6847-6856.

Shapiro, R., Fox, E. A. \& Riordan, J. F. (1989). Biochemistry, 28, 1726-1732.

Shapiro, R. \& Vallee, B. L. (1989). Biochemistry, 28, 7401-7408.

Shapiro, R. \& Vallee, B. L. (1992). Biochemistry, 31, $12477-$ 12485.

Strydom, D. J. (1998). Cell. Mol. Life Sci. 54, 811-824.

Strydom, D. J., Fett, J. W., Lobb, R. R., Alderman, E. M., Bethune, J. L., Riordan, J. F. \& Vallee, B. L. (1985). Biochemistry, 24, 54865494.
Tarragona-Fiol, A., Eggelte, H. J., Harbron, S., Sanchez, E., Taylorson, C. J., Ward, J. M. \& Rabin, B. R. (1993). Protein Eng. 6, 901-906.

Toiron, C., Gonzales, C., Bruix, M. \& Rico, M. (1996). Protein Sci. 5, 1633-1647.

Tsuji, T., Sun, Y., Kishimoto, K., Olson, K. A., Liu, S., Hirukawa, S. \& Hu, G.-F. (2005). Cancer Res. 65, 1352-1360.

Vaguine, A. A., Richelle, J. \& Wodak, S. J. (1999). Acta Cryst. D55, 191-205.

Wlodawer, A., Miller, M. \& Sjolin, L. (1983). Proc. Natl Acad. Sci. USA, 80, 3628-3631.

Wlodawer, A. \& Sjölin, L. (1983). Biochemistry, 22, 2720-2728.

Zegers, I., Maes, D., Dao-Thi, M.-H., Poortmans, F., Palmer, R. \& Wyns, L. (1994). Protein Sci. 3, 2322-2339. 